\title{
Zina percutaneous screw fixation combined with endoscopic lumbar intervertebral fusion under intraoperative neuromonitoring: a technique note report
}

\author{
Tong Yu \\ Jilin University Second Hospital \\ Jiu-Ping Wu \\ Jilin University Second Hospital \\ Jun Zhang \\ Jilin University Second Hospital \\ Hai-Chi Yu \\ Jilin University Second Hospital \\ Tian-Yang Yuan \\ Jilin University Second Hospital \\ Hai-Qing Tian \\ Jilin University Second Hospital \\ Su-Li Luo \\ Jilin University Second Hospital \\ Qin-Yi Liu ( $\nabla$ qinyi@jlu.edu.cn ) \\ Jilin University Second Hospital https://orcid.org/0000-0003-2700-1305
}

Technical advance

Keywords: endoscopic, lumbar intervertebral fusion, lumbar disc herniation, percutaneous, minimally invasive

Posted Date: July 30th, 2019

DOI: https://doi.org/10.21203/rs.2.12095/v1

License: (c) (i) This work is licensed under a Creative Commons Attribution 4.0 International License. Read Full License 


\section{Abstract}

Abstract Background: Open discectomy and intervertebral fusion surgery, including posterior lumbar intervertebral fusion (PLIF), anterior lumbar intervertebral fusion (ALIF), oblique lateral lumbar intervertebral fusion (OLIF), transforaminal lumbar intervertebral fusion (TLIF), and direct lateral lumbar intervertebral fusion (DLIF), are the common strategies for lumbar disc herniation (LDH), but they require quite a long recovery period. Zina percutaneous screw fixation combined with endoscopic lumbar intervertebral fusion (ZELIF) has the advantages in quicker recovery, less soft tissue destruction, shorter hospital stays, and less pain. We report a novel technique of ZELIF under intraoperative neuromonitoring (INM) for the treatment of LDH. Methods: a 51-year-old male with left lower extremity pain and numbness for 1 year was diagnosed with lumbar disc herniation (LDH). This patient was treated with Zina percutaneous screw fixation combined with endoscopic neural decompression, endplate preparation, and intervertebral fusion through Kambin's triangle. Each step of the operation was performed under INM. Results: The follow-up period lasted 1 months; the hospitalization lasted 4 nights; the blood loss volume was $65 \mathrm{ml}$, and the time of operation was 266 minutes. INM showed no neurological damage during the surgery. No surgical complications, including neurological deterioration, cage migration, nonunion, instrumentation failure or revision operation, were observed during the follow-up period. Visual Analogue Scale (VAS) score reduced from 7 to 1 ; the Oswestry Disability Index (ODI) decreased from 43 to 14; the EQ-5D score was 10 preoperatively and 15 at the final follow-up visit; the Physical Component Summary of the 36-Item Short Form Health Survey (SF-36) was 48 preoperatively and 49 at the last follow up visit; the SF-36 Mental Component Summary was 47 before surgery and decreased to 41 postoperatively. Conclusion: ZELIF under INM may represent a feasible, safe and effective alternative to endoscopic intervertebral fusion and percutaneous screw fixation, for decompressing the lumbar's exiting nerve root directly with minimal invasion in selected patients.

\section{Background}

In selected patients, spinal fusion is a procedure that has been proven to effectively improve the quality of life and significantly relieve pain [1,2]. Various strategies can be used in lumbar intervertebral fusion (LIF), including ALIF, PLIF, OLIF, TLIF, and DLIF. ALIF can provide adequate decompression of ventral dura, but it is not suitable for L4-5 and L5-S1 segments. PLIF can provide adequate decompression of the dorsal dural, but it is a relatively wide open surgery and hence has the disadvantage of significant pain and long recovery time.

The application of tubular dilators for nerve root decompression, combined with percutaneous screws and specialized internal cages, makes it possible to replace open fusion surgery with minimally invasive surgery. However, a lot of intervertebral fusion surgeries minimally invasive still need a muscle-tissue incision to insert a tube. The technique of transforaminal endoscopy can achieve the minimally invasive decompression, and numerous endoscopic LIF strategies have been reported for LDH [3-7]. However, various endoscopic LIF techniques have shortcomings, particularly in the placement of an established rigid bullet-shaped cage which is too large to pass through the endoscopic working channel. 
Some surgical instruments (the square channel with C-shaped opening and the square channel clamp) were modified so that the endoscopic LIF could be completed easily. ZELIF has many advantages, such as allowing the traditional size cage insertion, safe nerve root decompression, a small volume of bleeding, short hospitalization time and rapid recovery. Here, a report on the steps, matters for attention, benefits, indications, and contraindications of the ZELIF technique is given.

\section{Materials And Methods}

\subsection{Patient characteristics}

Examination revealed the significant lateral hypoesthesia of the left leg. The muscle strength of quadriceps femoris was at Grade V; the muscle strength of the left tibialis anterior was at Grade IV; the muscle strength of the left peroneus longus was at Grade III; the muscular tension of extremities, and the tendon reflex of the bilateral knees and bilateral Achilles were normal.

Magnetic resonance imaging (MRI) demonstrated LDH (L4-5) (Fig. 1A-B). The 3D CT of the lumbar spine showed straight lumbar curvature, lumbar osteoporosis, and L4-5 disc herniation. The lumbar vertebrae Xray film displayed that the curvature of the lumbar spine became straight. Electromyography of lower extremities illustrated that the peroneus longus muscle and the tibialis anterior muscle were subject to neurogenic damage. The patient was diagnosed primarily with LDH (L4-5).

The surgical segment, operation time, hospitalization time, and surgical complications were recorded. VAS score, ODI score, SF-36 score, and EQ-5D were evaluated.

\subsection{Anesthesia and INM techniques}

General anesthesia with intubation was achieved using propofol $(200 \mathrm{mg} / \mathrm{kg})$, fentanyl $(250 \mathrm{mg})$, and midazolam ( $2 \mathrm{mg})$. In addition, propofol $(0.2-0.5 \mathrm{mg} / \mathrm{kg}$ per hour) was constantly infused for maintaining anesthesia. Short-acting muscle relaxants with succinylcholine $(1 \mathrm{mg} / \mathrm{kg})$ were only provided during induction and intubation. The intraoperative nerve monitoring technique from Yu et al. [8,9] was applied in this surgery.

\subsection{Operative technique}

1. The patient was positioned prone on an operating table after general anesthesia (Fig. 2).

2. The needle of Zina percutaneous pedicle screw (Shanghai Sanyou Medical Devices Co., Ltd. China) was inserted. If the intervertebral space was too small, it should be distracted; if the intervertebral space was not narrow, it should not be distracted.

3. Endoscope implantation (Medical Intervertebral Endoscopic Surgery System, Shenzhen Shenzhou Medical Equipment Co., Ltd. China) was implemented. The endoscopic incision was located at 2-3 transverse fingers outside the pedicle and 50-60 degrees from the horizontal plane. The endoscopic saw was used to remove part of the superior articular process of L5 vertebral body to enlarge the 
intervertebral foramen (Fig. 3a-d) and complete the arthroplasty of the superior articular process of L5 vertebral body. Note: the superior articular process should not be too large in case of massive bleeding.

4. Nerve root decompression was performed under endoscopic (Fig. 4), and all endoscopic tools were removed after decompression.

5. ZELIF tools (Shanghai Sanyou Medical Devices Co., Ltd. China) (Fig. 5a-f) were installed. The key parts of ZELIF tools are the expandable square channel with C-shaped opening and the square channel clamp, the main function of which is to simplify the installation of the cage device. ZELIF incision was performed using the same method as that of endoscope incision. The guide needle was placed under fluoroscopy. The direction of the guide needle was parallel to the vertebral endplate on the lateral radiograph, and the tip of the needle was located in the center of the intervertebral space on the anteroposterior radiograph. The square canula was inserted into the intervertebral space along the direction of the guide needle, and then a square channel with C-shaped opening was inserted into the intervertebral space along the direction of the square sleeve; the square canula and the guide needle were taken out; at last the square channel clamp was installed to complete the establishment of the channel. Note: The square channel must be placed in the Kambin's triangle [10] to avoid nerve root injury. II. The functional status of the nerve root should be monitored during operation.

6. Intervertebral space management (Fig. 6). The intervertebral disc tissue was removed with pliers, a scraping spoon, a triangular scraping spoon, a scraper, and a reamer.

7. According to the test model, the cage with an appropriate size was selected and then inserted (Fig.

7a-e). The position of the cage was confirmed by fluoroscopy, and the square channel was taken out.

8. Endoscopic exploration of the nerve root and the cage (Fig. 8).

9. A Zina screw was inserted and a titanium rod was installed (Fig. 9a-b).

\section{Results}

The follow-up period lasted 1 months; the hospitalization lasted 4 nights. The blood loss volume was 65 $\mathrm{ml}$, and the time of operation was 266 minutes. INM showed no neurological damage during surgery. No surgical complications, such as neurological deterioration, cage migration or nonunion, were found during the follow-up period (Table 1). The VAS score reduced from 7 to 1 ; the ODI score decreased from 43 to 14; the EQ-5D score was 10 preoperatively and 15 at the final follow-up visit; the SF-36 Physical Component Summary was 48 preoperatively and 49 at the last follow up visit; and the SF-36 Mental Component Summary was 47 before surgery and decreased to 41 postoperatively (Table 2).

\section{Discussion}

Compared with other surgical disciplines including open surgery and sports medicine in orthopedics, endoscopic minimally invasive surgery has been considered to be a sensible method that satisfies the 
demands of the quicker recovery for LDH in recent years [7]. Minimally invasive spinal surgery is gaining popularity because its several advantages, including restoring functions while preserving normal anatomy, minimizing hospitalization and complications associated with extensive open procedures, and helping elderly patients return to active premorbid status as early as possible [6]. Endoscopic lumbar intervertebral fusion has been reported. Since the incidence of complications is as high as $36 \%$, it is not recommended by Jacquot et al [5]. However, ZWLIF technique can finish lumbar intervertebral fusion and percutaneous screw fixation safely without technical limitations. To the best of our knowledge, no study on ZELIF has been reported yet. Thus, we intended to share a relevant technical note.

Numerous prevenient studies have discussed the anatomical structure of intervertebral foramina in the lumbar spine, so as to measure the maximum working channel space suitable for undergoing endoscopic discectomy manipulations [11-14]. In 1995, Mirkovic et al. [13] reported the size of the safe edge for a channel in the intervertebral foramen. In 2005, Min et al. [12] demonstrated that the average distance between the superior articulating process and the exiting nerve root was $11.6 \mathrm{~mm}$. In 2016, from a cadaveric study, Hardenbrook $\mathrm{M}$ et al. [15] reported a relatively large area in the lumbar intervertebral foramen, called Kambin's triangle. Therefore, we believe that the ZELIF channel is safe to be installed through Kambin's triangle, and this idea has been agreed by Ozer et al. [10]. Although it is theoretically safe, INM is carried out to prevent nerve injury.

Indications of this technique include (1) LDH with segmental instability, (2) lumbar spinal stenosis with segmental instability and (3) lumbar spondylolisthesis of lower than Meyerding grade II.

Contraindications mainly include (1) L5-S1 lumbar disc herniation due to the high ilium which affects the installation of the channel, (2) variation of the nerve root, (3) lumbar spondylolisthesis of higher than Meyerding grade III, and (4) severe central canal stenosis.

Advantages of ZELIF include shorter operation time, less blood loss, shorter hospital stays, a very low risk for pulmonary embolism, less soft tissue destruction, less pain, quicker restoring the function of standing and walking, and no need for drain postoperatively [5]. In addition, the decompression of dura and the nerve root was performed under endoscope and INM, avoiding nerve injury and improving the safety of operation.

Literature reports suggest that smaller fusion cages must be used when endoscope-guided intrabody cages are placed, because traditional fusion cages are too large to pass through working channels $[5,16]$. However, the undersized cage may directly cause cage migration into the intervertebral foramen or spinal canal, thus leading to neurological compromise, failure of fixation, or even revision operation $[17,18]$. The ZELIF technology uses a specially designed C-shaped open channel, which can realize the installation of conventional cages under fluoroscopy without size reduction. In this study, cage migration was not observed during 1 months of the follow-up, and we attribute this positive result to the ZELIF technique.

INM has been commonly utilized to avoid nerve damage in spinal surgery $[9,19]$, and considered by many scholars as a reliable method to avoid nerve injury $[9,19,20]$. Even if there are no neuromonitoring events during the operation, we believe that it is necessary to monitor the functional state of the nerve root, 
especially in the process of channel installation. We suggest that once abnormal nerve monitoring is found, the installation direction of the channel should be adjusted in time.

The following points should be paid attention to during the operation: (1) After the establishment of the channel, it is suggested that the assistant should fix the channel by hand to avoid it sliding out due to improper operation; (2) If the channel is taken out accidentally, please re-enter the guide wire and re-install the channel. Blind direct installation of the channel is forbidden; (3) Careful examination of the patients should be performed preoperatively, and nerve root variation is not suitable for ZELIF; (4) INM is recommended during all surgical steps.

\section{Conclusion}

ZELIF under INM may represent a feasible, safe and effective alternative to endoscopic intervertebral fusion and percutaneous screw fixation with minimal invasive in selected patients for decompressing the exiting nerve root of the lumbar directly.

\section{Abbreviations}

ZELIF: Zina percutaneous screw fixation combined with endoscopic lumbar intervertebral fusion; INM: Intraoperative neuromonitoring; LDH: Lumbar disc herniation; ALIF: Anterior lumbar intervertebral fusion; PLIF: Posterior lumbar intervertebral fusion; DLIF: Direct lateral lumbar intervertebral fusion; OLIF: Oblique lateral lumbar intervertebral fusion; TLIF: transforaminal lumbar intervertebral fusion; SSEPs:

Somatosensory evoked potentials; TcMEP: Transcranial motor evoked potentials; TEMG: Triggered electromyography.

\section{Declarations}

\section{Acknowledgements}

We gratefully acknowledge the cooperation of the doctors and nurses in the operating room.

\section{Ethical approval and consent to participate}

Patient has provided informed consent for publication of the case. This report was approved by the ethics committee of the Second Hospital of Jilin University, Changchun, China.

\section{Consent for publication}

We have obtained consent to publish from the participant to report individual patient data. 


\section{Availability of data and materials}

The datasets used during the current study are available from the corresponding author on reasonable request.

\section{Competing interests}

There were no competing interests in this study.

\section{Funding}

No funding was provided for this study.

\section{Authors' contributions}

Conceptualization: Tian-Yang Yuan, Jun Zhang.

Data curation: Jiu-Ping Wu.

Investigation: Hai-Chi Yu, Tian-Yang Yuan.

Methodology: Tong Yu, Qin-Yi Liu.

Project administration: Su-Li Luo, Hai-Qing Tian.

Supervision: Qin-Yi Liu.

Writing - original draft: Tong Yu, Jun Zhang.

Writing- review \& editing: Qin-Yi Liu.

\section{References}

1. Gan F, Jiang J, Xie Z, Huang S, Li Y, Chen G, Tan H: Minimally invasive direct lateral interbody fusion in the treatment of the thoracic and lumbar spinal tuberculosisMini-DLIF for the thoracic and lumbar 
spinal tuberculosis. BMC Musculoskelet Disord 2018, 19(1):283.

2. Pearson AM, Lurie JD, Tosteson TD, Zhao W, Abdu WA, Weinstein JN: Who should undergo surgery for degenerative spondylolisthesis? Treatment effect predictors in SPORT. Spine (Phila Pa 1976) 2013, 38(21):1799-1811.

3. Wang MY, Grossman J: Endoscopic minimally invasive transforaminal interbody fusion without general anesthesia: initial clinical experience with 1-year follow-up. Neurosurg Focus 2016, 40(2):E13.

4. Heo DH, Son SK, Eum JH, Park CK: Fully endoscopic lumbar interbody fusion using a percutaneous unilateral biportal endoscopic technique: technical note and preliminary clinical results. Neurosurg Focus 2017, 43(2):E8.

5. Jacquot $F$, Gastambide D: Percutaneous endoscopic transforaminal lumbar interbody fusion: is it worth it? Int Orthop 2013, 37(8):1507-1510.

6. Osman SG: Endoscopic transforaminal decompression, interbody fusion, and percutaneous pedicle screw implantation of the lumbar spine: A case series report. Int J Spine Surg 2012, 6:157-166.

7. Youn MS, Shin JK, Goh TS, Lee JS: Full endoscopic lumbar interbody fusion (FELIF): technical note. Eur Spine J 2018, 27(8):1949-1955.

8. Yu T, Li QJ, Zhang XW, Wang Y, Jiang QY, Zhu XJ, Jiang ZD, Zhao JW: Multimodal intraoperative monitoring during surgical correction of scoliosis to avoid neurologic damage. Medicine (Baltimore) 2019, 98(15):e15067.

9. Yu T, Wang Y, Zhang XW, Jiang ZD, Zhu XJ, Jiang QY, Zhao JW: Multimodal intraoperative monitoring during reduction of spine burst fracture and dislocation prevents neurologic injury. Medicine (Baltimore) 2018, 97(10):e0066.

10. Ozer AF, Suzer T, Can H, Falsafi M, Aydin M, Sasani M, Oktenoglu T: Anatomic Assessment of Variations in Kambin's Triangle: A Surgical and Cadaver Study. World Neurosurg 2017, 100:498-503.

11. Arslan M, Comert A, Acar HI, Ozdemir M, Elhan A, Tekdemir I, Tubbs RS, Ugur HC: Nerve root to lumbar disc relationships at the intervertebral foramen from a surgical viewpoint: An anatomical study. Clin Anat 2012, 25(2):218-223.

12. Min JH, Kang SH, Lee JB, Cho TH, Suh JK, Rhyu IJ: Morphometric analysis of the working zone for endoscopic lumbar discectomy. J Spinal Disord Tech 2005, 18(2):132-135.

13. Mirkovic SR, Schwartz DG, Glazier KD: Anatomic considerations in lumbar posterolateral percutaneous procedures. Spine (Phila Pa 1976) 1995, 20(18):1965-1971.

14. Xin G, Shi-Sheng H, Hai-Long Z: Morphometric analysis of the YESS and TESSYS techniques of percutaneous transforaminal endoscopic lumbar discectomy. Clin Anat 2013, 26(6):728-734.

15. Hardenbrook M, Lombardo S, Wilson MC, Telfeian AE: The anatomic rationale for transforaminal endoscopic interbody fusion: a cadaveric analysis. Neurosurg Focus 2016, 40(2):E12.

16. He EX, Guo J, Ling QJ, Yin ZX, Wang Y, Li M: Application of a narrow-surface cage in full endoscopic minimally invasive transforaminal lumbar interbody fusion. Int J Surg 2017, 42:83-89. 
17. Hu YH, Niu CC, Hsieh MK, Tsai TT, Chen WJ, Lai PL: Cage positioning as a risk factor for posterior cage migration following transforaminal lumbar interbody fusion - an analysis of 953 cases. $B M C$ Musculoskelet Disord 2019, 20(1):260.

18. Aoki Y, Yamagata M, Nakajima F, Ikeda Y, Takahashi K: Posterior migration of fusion cages in degenerative lumbar disease treated with transforaminal lumbar interbody fusion: a report of three patients. Spine (Phila Pa 1976) 2009, 34(1):E54-58.

19. Thirumala PD, Huang J, Thiagarajan K, Cheng H, Balzer J, Crammond DJ: Diagnostic Accuracy of Combined Multimodality Somatosensory Evoked Potential and Transcranial Motor Evoked Potential Intraoperative Monitoring in Patients With Idiopathic Scoliosis. Spine (Phila Pa 1976) 2016, 41(19):E1177-1184.

20. Hu Y, Luk KD, Lu WW, Leong JC: Application of time-frequency analysis to somatosensory evoked potential for intraoperative spinal cord monitoring. J Neurol Neurosurg Psychiatry 2003, 74(1):82-87.

\section{Figures}

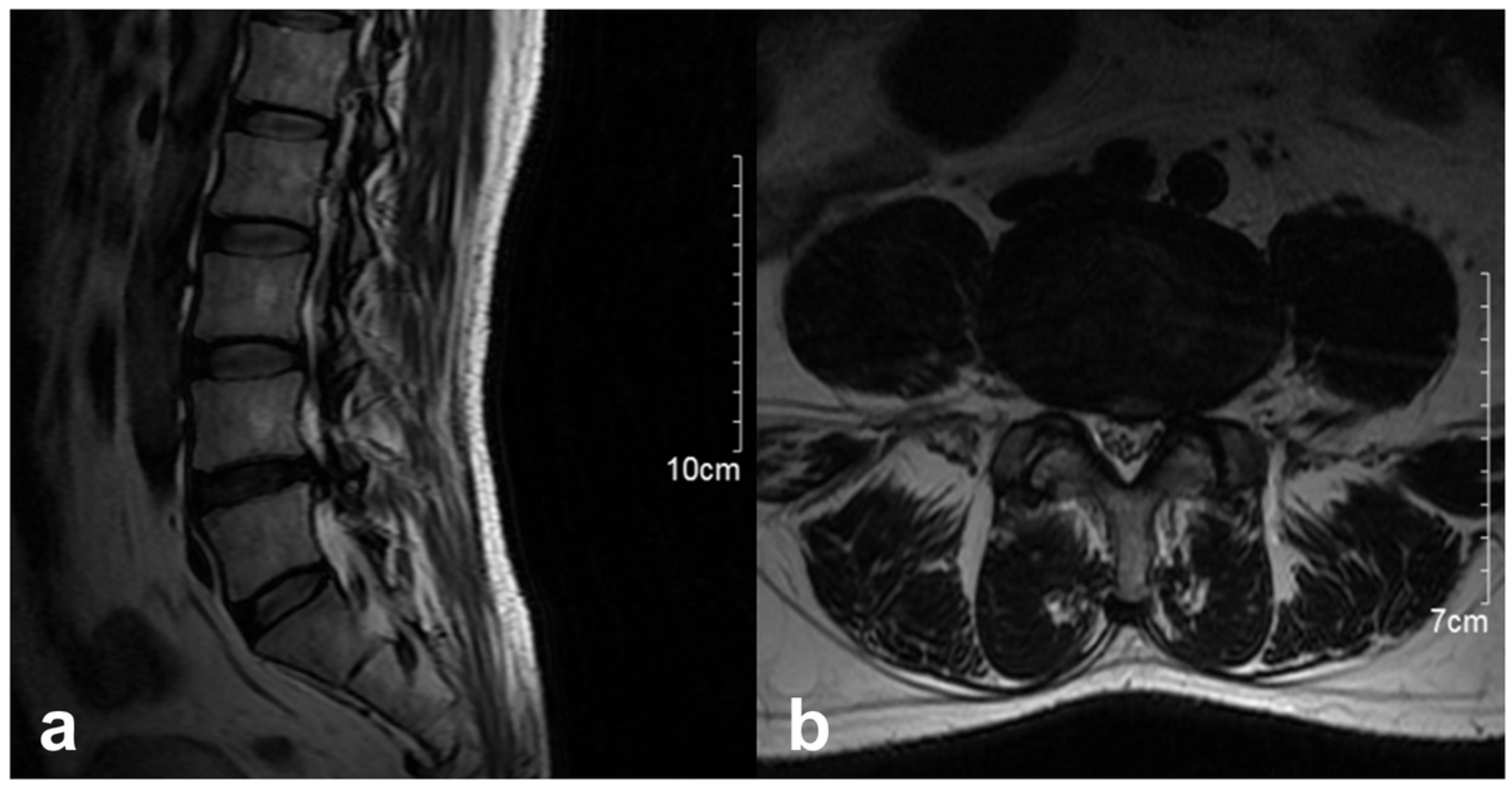

Figure 1

The sagittal (a) and axial (b) of MRI showed LDH (L4-5).

展

Figure 2

The patient was placed in a prone position. 


\section{Figure 3}

The yellow triangle represents Kambin's triangle and the bone in the red circle is part of the L5 that needs to be resected (a), the blue, yellow and red regions represent L4 inferior articular process and L5 superior articular process after partial resection (b), anteroposterior (c) and lateral (d) radiographs of the L5 superior articular process arthroplasty.

$$
\text { त्ये }
$$

\section{Figure 4}

Nerve root decompression was conducted under endoscopic. The red arrow represents the nerve root; the yellow arrow represents the endplate and the green arrow represents the intervertebral space after decompression.
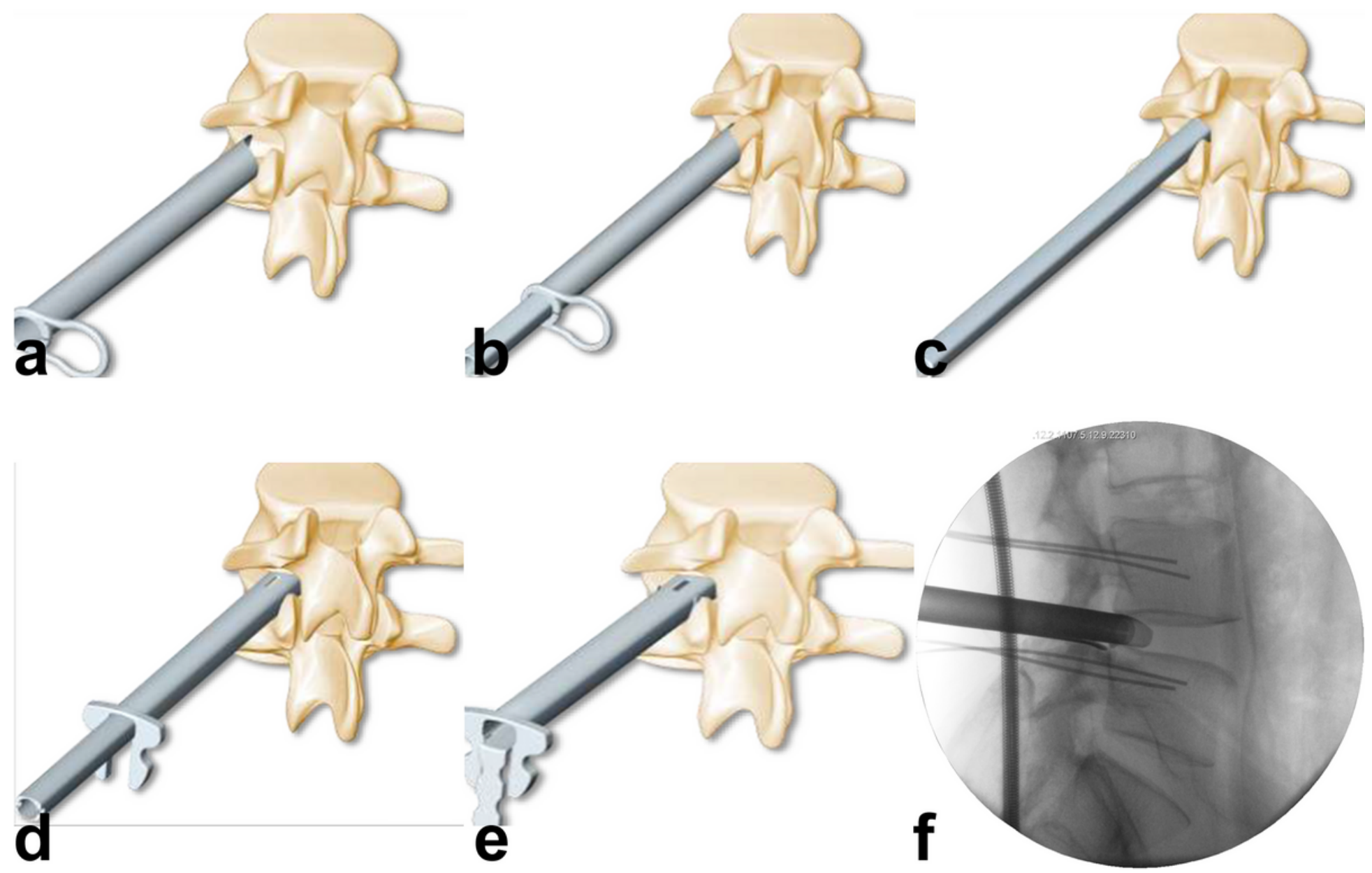

\section{Figure 5}

Round canula implantation (a), square canula insertion (b), round canula (c) was taken out); the square channel with C-shaped opening (d) was inserted; the square channel clamp (e) was installed; the lateral 
radiograph (f) showed that the establishment of the channel was completed successfully.

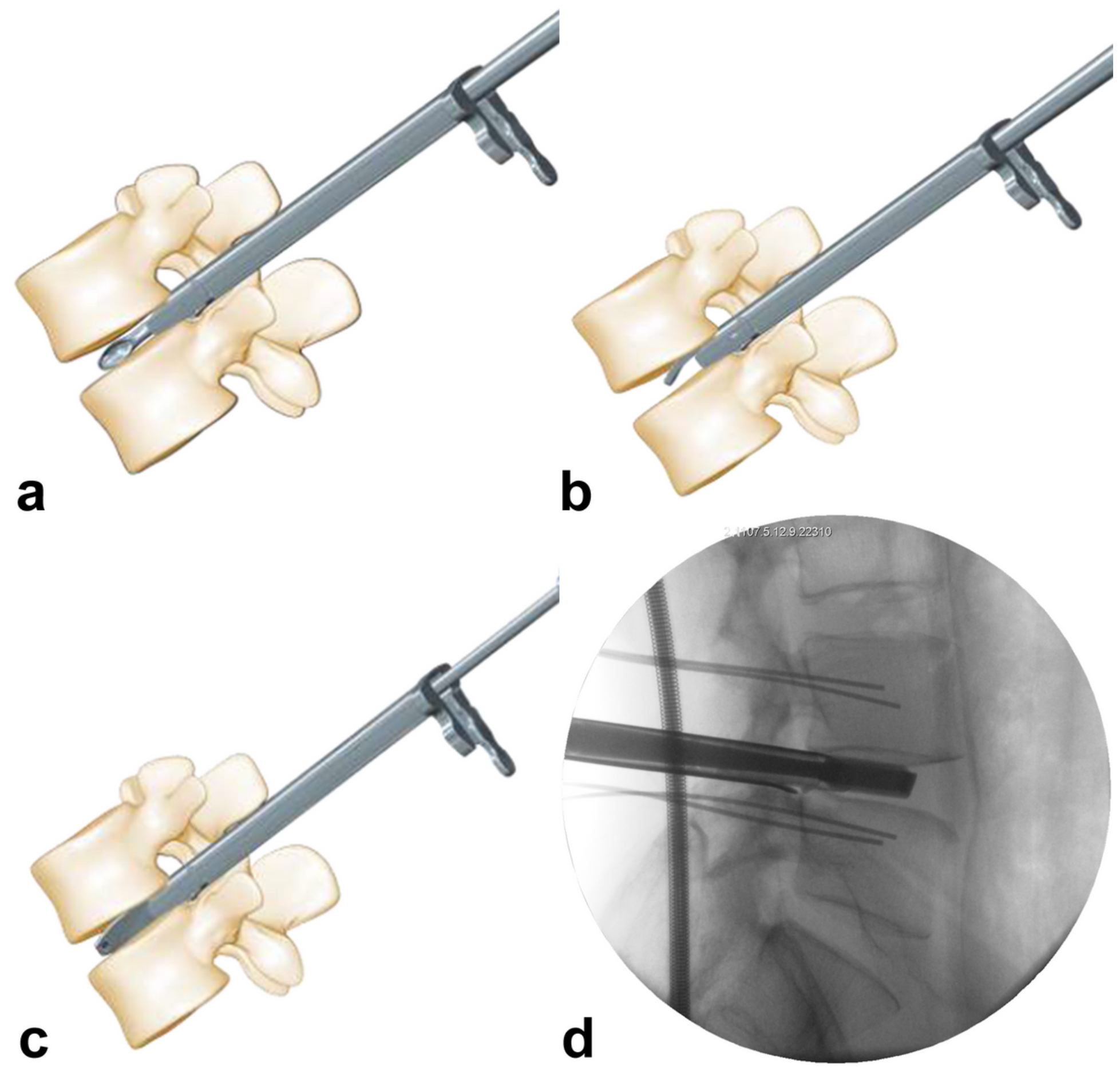

Figure 6

The intervertebral disc tissue was removed with a scraping spoon (a), a triangular scraping spoon (b), and a scraper (c and d).

原

Figure 7 
According to the test model ( $a$ and $b$ ), the appropriate size of the cage was selected, and the position of the cage was examined by anteroposterior (c) and lateral (d) radiographs.

娄

\section{Figure 8}

Endoscopic observation of the cage that indicated by blue arrows.

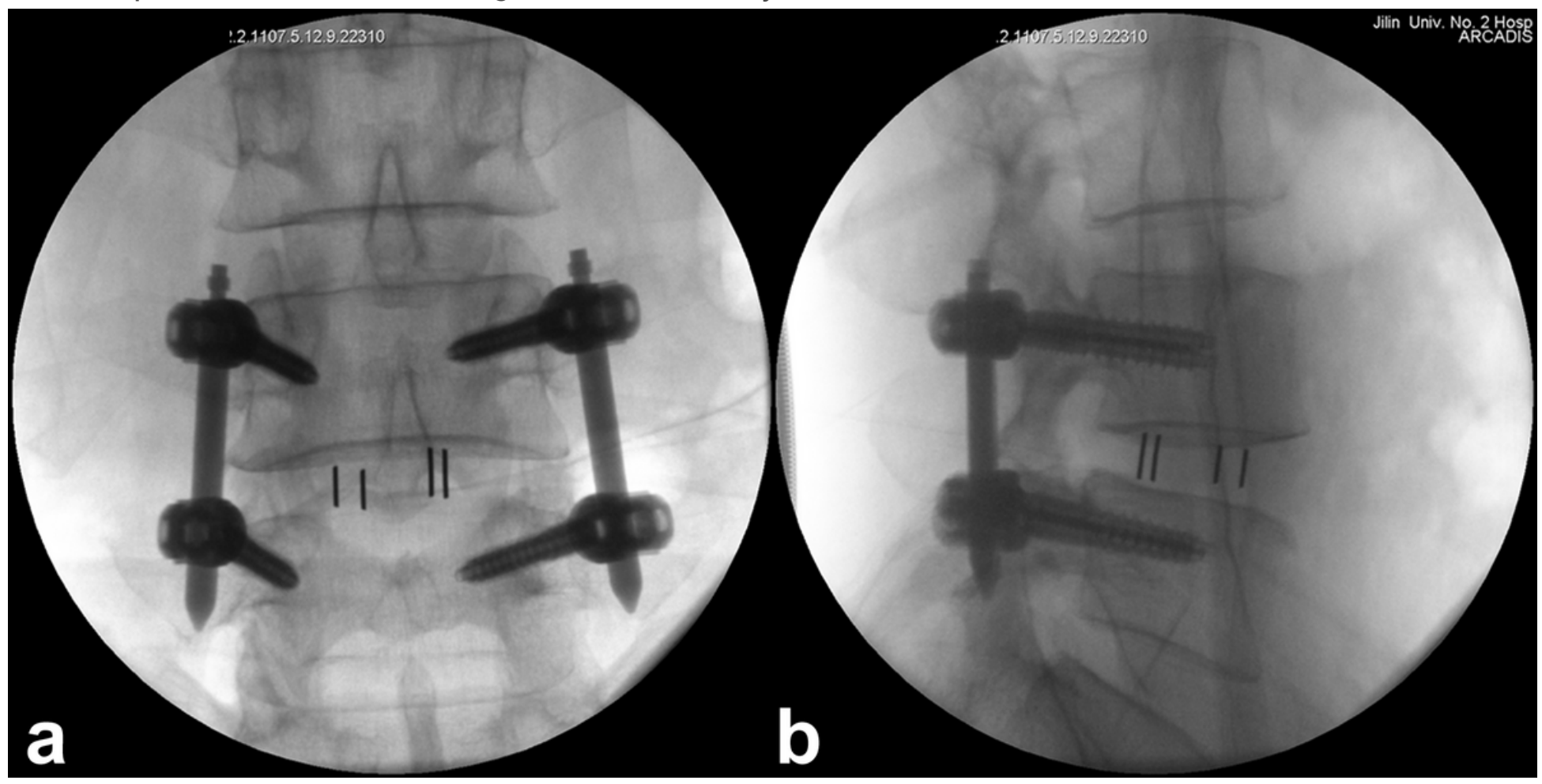

Figure 9

Anteroposterior (a) and lateral (b) radiographs were taken postoperatively and showed that the Zina percutaneous pedicle screws and the cage were in a good position.

\section{Supplementary Files}

This is a list of supplementary files associated with this preprint. Click to download.

- Table2.pdf

- Table1.pdf 